\title{
Molecular dynamics studies of homogeneous nucleation in solid-state transitions
}

\author{
Shimin Xu and Lawrence S. Bartell \\ Department of Chemistry, University of Michigan, Ann Arbor, MI 48109 \\ Received 16 September 1992
}

\begin{abstract}
Simulations of clusters containing 100 to 250 molecules of $\mathrm{TeF}_{6}$ successfully reproduce the crystalline packing arrangements observed in electron diffraction investigations of large molecular clusters ( $\sim 10^{4}$ molecules) of the same material. More remarkably, when the clusters are cooled step by step in MD computations at a rate of ca.

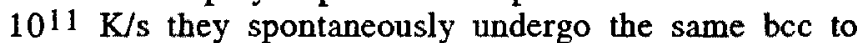
monoclinic phase transition that has been observed experimentally in supersonic flow, despite the million-fold difference in the timescales involved. The existence of such a correspondence over so many orders of magnitude, in itself, imposes severe constraints on what type of molecular mechanism can underlie the transformation. Even more revealing evidence about the molecular behavior associated with the phase change is provided by the simulations. They show the formation of the actual transition complexes along the transition pathway, namely, the critical nuclei of the new phase. These nuclei, which are made up of approximately 20 molecules, can be recognized in the midst of the surrounding matter. Techniques based on molecular orientations, involving Pawley-Fuchs projections and orientational angular distribution functions, make it possible to estimate the size of critical nuclei. One noteworthy result established in the simulations is that the solid-state transition temperature from bcc to monoclinic depends upon particle size in the same manner as does the freezing point.
\end{abstract}

PACS: $36.40 ; 64.60 . \mathrm{Qb} ; 64.70 . \mathrm{Kb} ; 82.20 . \mathrm{Wt}$

\section{Introduction}

The subject of the kinetics of phase transitions in condensed matter is important not only in science, but also in technology. Fundamental information about phase changes in solid materials has proven to be particularly difficult to obtain. Most of the work on transformations between crystalline phases has focused upon the overall rate of transformation rather than upon the nucleation rate itself.
Recently, Dibble and Bartell showed that it is possible to measure the nucleation rates for solid state phase transitions by diffracting electrons from undercooled molecular clusters in a supersonic jet [1]. Interpreting their results in terms of classical nucleation theory, they showed for the cases examined that the mechanisms were consistent with the reorientation of molecules and they determined the corresponding interfacial free energies between the two crystalline phases concerned.

Although diffraction experiments have provided useful new information about the structural chemistry of condensing aggregates, the diffraction technique is unable to follow the molecular behavior in the very early stages of phase changes. Moreover, it is difficult in such experiments to determine the transition temperature. On the other hand, molecular dynamics simulations are able to provide detailed accounts of atomic and molecular behavior during phase transitions, including the nucleation process itself [2].

Therefore, it seemed worthwhile to initiate computer simulations of small clusters in an effort to shed light on the unseen molecular activity. Tellurium hexafluoride appeared to be an attractive system to examine because it exhibits interesting phase transitions [3], its constituent molecules are simple and symmetrical with fairly well known intermolecular interactions, and it is closely related to $\mathrm{SeF}_{6}$ for which nucleation rates had been determined by electron diffraction [1].

\section{Computational details}

Molecular dynamics simulations were carried out on nearly spherical clusters of 100 to $250 \mathrm{TeF}_{6}$ molecules. Molecules were assumed to be rigid octahedra, interacting by pairwiseadditive atom-atom Lennard-Jones potential functions. The Lennard-Jones parameters have been tabulated elsewhere [3]. The program used was a modified version of the program MDMPOL from the SERC Daresbury Laboratory. Newton's equations of motion for centers of mass and for rotations were integrated by Verlet's leapfrog algorithm [4] with a step size of $10 \mathrm{fs}$. 
The simulation processes were as follows. Starting from the bcc configuration at a certain bath temperature (150 or $160 \mathrm{~K}$, at which temperatures the bcc phase of the clusters is stable), an initial bath temperature was maintained by rescaling each of the first 1000 timesteps. After this period of equilibration the temperature rescaling was switched off and constant energy MD trajectories were followed for 4000 timesteps during which thermodynamic averages such as potential energy and temperature were calculated, and coordinates, quaternion parameters, etc., of all the molecules were saved every 50 timesteps.

The bath temperature was dropped by $10^{\circ}$ after each period of 5000 timesteps, and the process was repeated with the current configuration and rescaled velocities and angular momenta. Runs were continued until the bath temperature reached $10 \mathrm{~K}$.

\section{Results}

When clusters of 100,150 , and 250 molecules were cooled down from the bcc phase, they all transformed to the monoclinic phase. The phase transition was readily recognized from the records of instantaneous configurational energies as a function of time. When the bcc clusters were cooled down, the 250-molecule cluster transformed to monoclinic at a bath temperature $90 \mathrm{~K}$, while the 150 - and 100 -molecule clusters transformed at $80 \mathrm{~K}$. Figure 1 shows the evolution of the instantaneous configurational energies for the 150-molecule cluster at bath temperatures of 90,80 ,

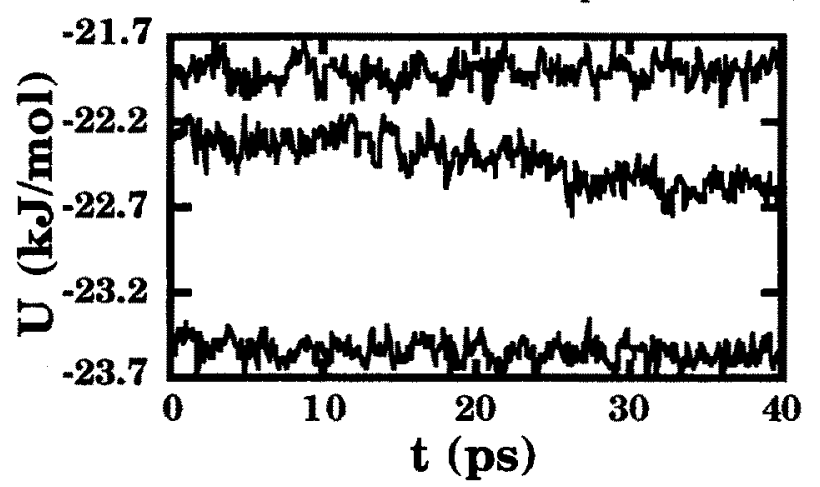

Fig. 1. Evolution of configurational energy of a 150-molecule cluster at bath temperatures of $90 \mathrm{~K}$ (upper), $80 \mathrm{~K}$ (middle), and $70 \mathrm{~K}$ (lower). Transition begins at $\sim 12 \mathrm{ps}$ (middle trace). and $70 \mathrm{~K}$.

The configuration which contains the critical nucleus was first discerned from the appearance of the record of instantaneous configurational energies. Once the time of appearance was identified approximately, it could be refined somewhat. A provisional configuration was selected, its instantaneous temperature was raised by 3 degrees, and a run at constant energy was carried out for 3000 timesteps. If no phase transition occurred, we took the configuration to be pre-critical. If one occurred, it was assumed the cluster contained either a critical, or a post-critical nucleus.

The size of critical nuclei could be determined approximately from the information implicit in the orientations of molecules in the clusters. Estimates could be based on Pawley-Fuchs projections [5] and from plots of the orientational angular distribution functions. Because the bcc and monoclinic phases are closely related translationally, we were unable to distinguish them by means of their Voronoi polyhedra [2]. The orientational angular distribution $P_{o}(\theta)$ is defined as

$P_{o}(\theta)=\frac{2}{N(N-1) N_{b}^{2}}\left\langle\sum_{i<j} \sum_{\alpha=1 \beta=1}^{N_{b}} \sum_{i=1}^{N_{b}} \delta\left[\theta-\left(\theta_{\alpha \beta}\right)_{i j}\right]\right)$

where $N$ is number of molecules in the cluster, $N_{b}$ is the number of bonds of the molecule, and $\left(\theta_{\alpha \beta}\right)_{i j}$ is the angle between bond $a$ on molecule $i$ and bond $b$ on molecule $j$, or

$$
\left(\theta_{\alpha \beta}\right)_{i j}=\cos ^{-1}\left[\left(\mathbf{n}_{\alpha}\right)_{i} \bullet\left(\mathbf{n}_{\beta}\right)_{j}\right]
$$

where $\left(\mathbf{n}_{\alpha)_{i}}\right.$ and $\left(\mathbf{n}_{\beta}\right)_{j}$ are the unit vectors along the bonds involved.

For the bcc phase, all molecules have the same orientation except for disorder. Therefore, their bond directions displayed in a Pawley-Fuchs projection show up as three patches of spots when viewed down a 3-fold axis. For the monoclinic phase, with one-third of its molecules shifted $60^{\circ}$ about the 3-fold axis, three additional patches appear whose individual spots identify the molecules belonging to their phase. In practice, estimating the number of molecules in the monoclinic phase requires a correction for the random background of spots associated with disordered surface molecules. An illustration of the evolution of a Pawley-Fuchs projection during the phase change from bcc
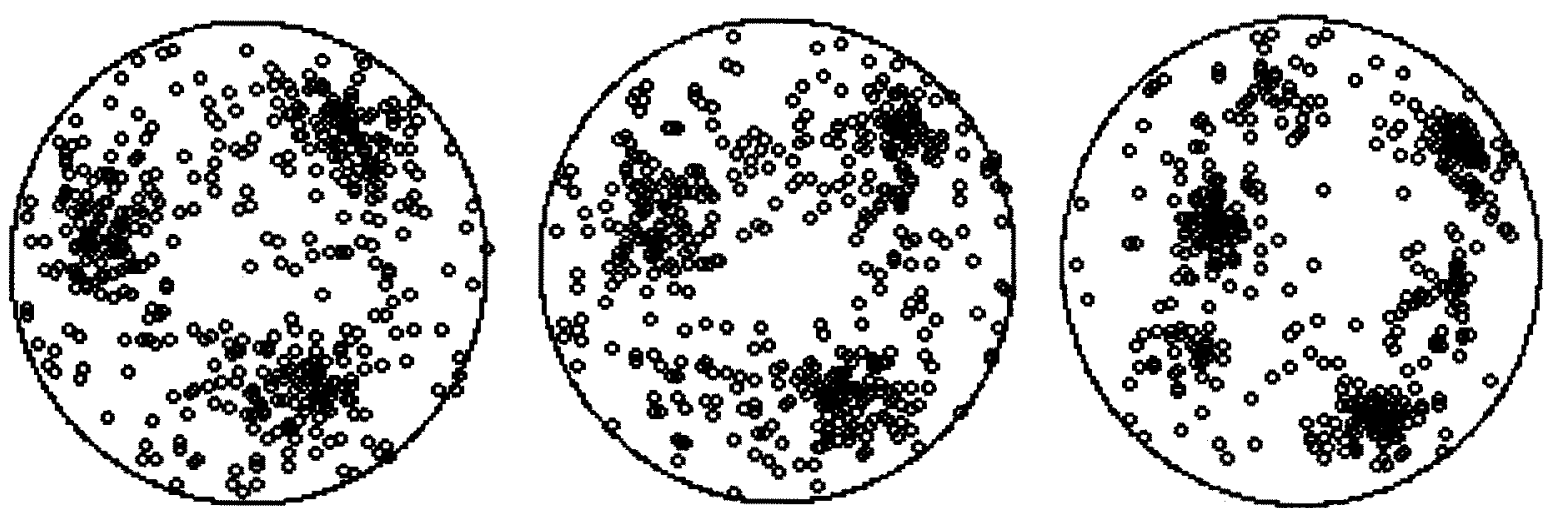

Fig. 2. Pawley-Fuchs projections of critical configuration (center), and the configurations at the end of runs at $90 \mathrm{~K}$ (left) and $80 \mathrm{~K}$ (right), for the 150-molecule cluster. 
(left) to monoclinic (right) in a 150 -molecule cluster is presented in Figure 2.

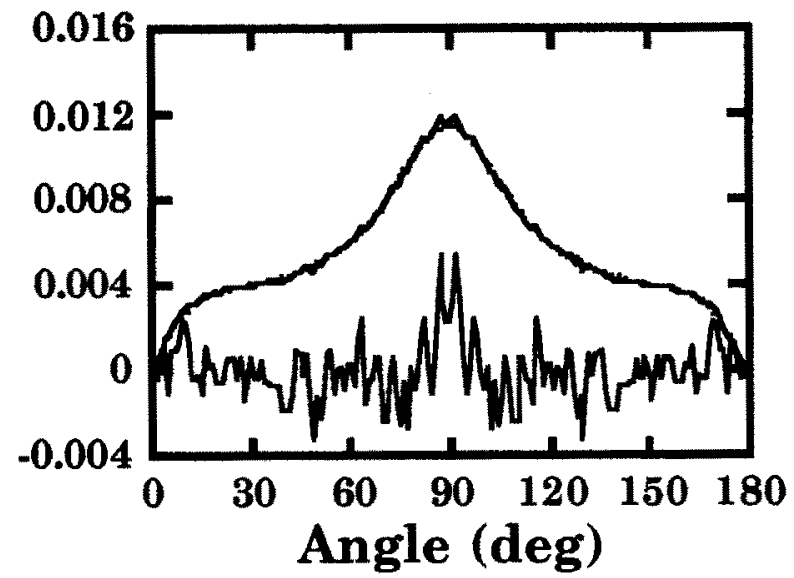

(a)

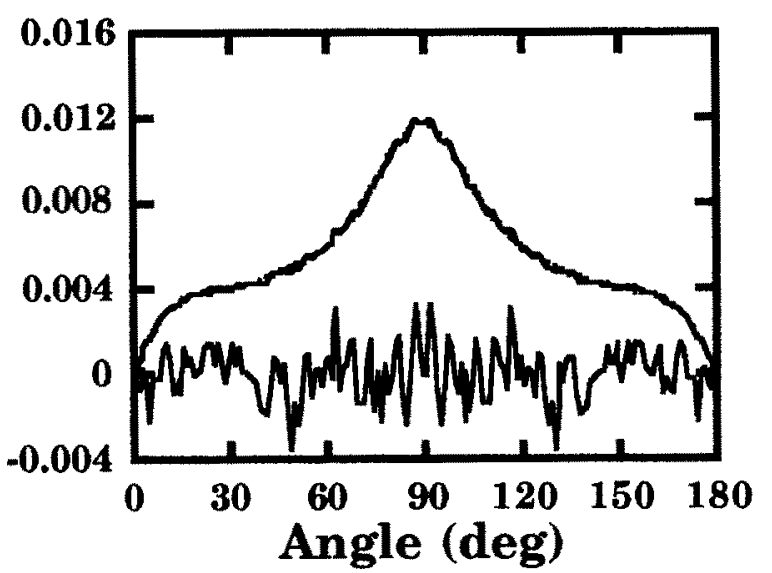

(b)

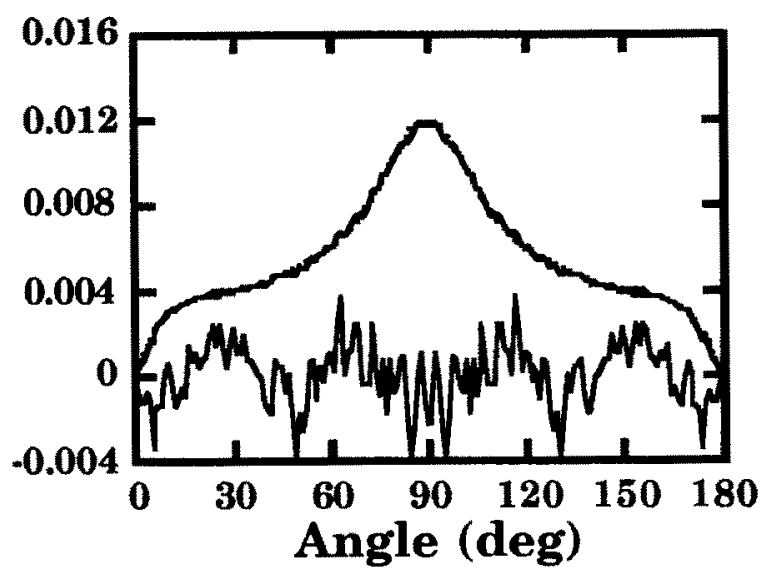

(c)
Results for the various clusters examined show that the transition tends to start in the interior of a cluster. At the critical configuration, roughly 4-8 of the molecules for the 100- and 150-molecule clusters, and 6-10 of the molecules for the 250-molecule cluster, have undergone a reorientation, indicating that the critical nucleus contains approximately 12-24 molecules for the 100- and 150-molecule clusters, and 18-30 molecules for the 250-molecule cluster.

For the 150-molecule cluster, the phase of the cluster is mostly bcc at $90 \mathrm{~K}$, while at $70 \mathrm{~K}$, it is mostly monoclinic. Figure 3 shows the orientational angular distribution of 50 configurations of the cluster during steepest-descent quenches, starting from the configuration containing the critical nucleus. For comparison are shown synthetic orientational angular distributions based on contributions of $3 \%, 7 \%$ and $11 \%$ from $70 \mathrm{~K}$ and $97 \%, 93 \%$, and $89 \%$ from $90 \mathrm{~K}$. Also shown is the difference between the actual and synthetic distributions. From these results we are able to estimate that the size of the critical nucleus is about 8-14 molecules for the cluster. From the orientational angular distributions for 100- and 250-molecule clusters we estimate that the critical nuclei contain about 11-17 and 25-31 molecules, respectively. Because the larger cluster is less undercooled, it would be expected to form a larger critical nucleus.

\section{Discussion}

This research appears to be the first molecular dynamics study of molecular clusters to obtain information about critical nuclei in a solid-state transition. Our results agree reasonably with those calculated from the classical theory of homogeneous nucleation if we use parameters extrapolated from those for $\mathrm{SeF}_{6}$ documented elsewhere [1]. They also show an effect of particle size on the transition temperature for a solid-state phase change that is analogous to the wellknown effect on the melting point. Such a similarity would be expected, in first order, if Turnbull's empirical relation for interfacial free energies [6] applied to boundaries between two solid phases as it does to those between solids and liquids. Although the results of our study are somewhat crude, they constitute a promising beginning in research to elucidate nucleation.

This research was supported by a grant from the National Science Foundation. We thank Dr. W. Smith of the Daresbury Laboratory for the program MDMPOL, and Mrs. M. Browning for professional formatting.

\section{References}

1. Dibble, T. S., Bartell, L. S.: J. Phys. Chem. in press

2. Swope, W. C., Andersen, H. C.: Phys, Rev. B 41, 7042 (1990)

3. Bartell, L. S., Xu, S.: J. Phys. Chem. 95, 8939 (1991)

4. Verlet, L.: Phys. Rev. 21, 165 (1968)

5. Fuchs, A. H., Pawley, G. S.: J. Phys. France 49, 41 (1988)

6. Turnbull, D.: J. Appl. Phys. 21, 1022 (1950) shown is $3 \%, 7 \%$, and $11 \%$ monoclinic, respectively. Also distributions, multiplied by a factor of 10 . 